\title{
BMP4 Gene
}

National Cancer Institute

\section{Source}

National Cancer Institute. BMP4 Gene. NCI Thesaurus. Code C95041.

This gene is involved in bone development and signaling. 